\title{
Trafficking Mechanism of Fungal Effector Proteins Inside Rice Cells
}

Chang Hyun Khang ${ }^{1}$, Elizabeth A. Richardson ${ }^{1}$, Yainitza Hernández-Rodríguez ${ }^{1}$, Li Hung Chen ${ }^{1}$, Timothy Todd ${ }^{2}$ and Barbara Valent ${ }^{2}$

1. Department of Plant Biology, University of Georgia, Athens, Georgia 30602, USA

2. Department of Plant Pathology, Kansas State University, Manhattan, Kansas 66506, USA

Rice blast disease, caused by the fungal pathogen Magnaporthe oryzae, is the most destructive disease of the world's rice crop, but strategies for durable disease control remain elusive. The rice blast fungus produces biotrophic invasive hyphae that successively invade living rice cells while separated from the rice cytoplasm by an extrainvasive hyphal membrane. To achieve biotrophic colonization, invasive hyphae secrete cytoplasmic effector proteins, which are preferentially localized in biotrophic interfacial complexes (BICs) and enter the cytoplasm of the rice cells, and apoplastic effector proteins, which remain in the host extracellular space [1, 2] (Figure 1). BICs are an infection-specific interfacial structure that are first formed in front of the tips of filamentous hyphae that enter rice cells, and then remain beside the first bulbous invasive hyphal cells. Cytoplasmic effector proteins, but not apoplastic effector proteins, continue to be secreted to BICs, suggesting BICs are a port for effector delivery into the host cell. BICs appear to contain aggregations of lamellar membranes immediately outside the fungal cell wall. These membranes were associated with an electron-dense region, presumed to be lipidrich, which appeared to be encapsulated by a cap-like structure (Figure 2).

In order to understand how cytoplasmic effector proteins are delivered into living rice cells, we focused on defining molecular motifs for accumulation in BICs and for effector translocation into the host cell. We produced and analyzed fungal transformants expressing chimeric genes between cytoplasmic effector genes, such as PWL2, and apoplastic effector genes, such as BAS4, coupled with live-cell fluorescence microscopy-based host translocation assays reported in Khang et al., 2010 (Figure 1). We have found that host translocation of cytoplasmic effector protein Pwl2p was significantly impaired when the PWL2 protein coding sequence was expressed with the upstream regulatory sequence of the apoplastic effector gene $B A S 4$. Impaired host translocation of the Pwl2p was correlated with the altered protein targeting pattern from preferential BIC-localization to the apoplastic hyphal outlining pattern, and also correlated with reduced avirulence activity. Consistent with the major role of the effector upstream regulatory sequence in determining protein localization specificity, apoplastic effector protein Bas $4 p$ was translocated into host cells when the protein coding sequence of the $B A S 4$ gene was expressed with the upstream regulatory sequence of the PWL2 gene. We have further determined that the upstream regulatory sequence and signal peptide-encoding sequence of effector genes are the minimal sequences that determine both preferential BIC localization and delivery to the rice cytoplasm or apoplastic space.

To determine if the expression of cytoplasmic effector genes was induced in BIC-associated cells, we developed a live-cell imaging-based assay for quantification of PWL2 effector gene expression. The PWL2 gene was strongly up-regulated in the BIC-associated cells. After the hyphae continued to grow, cytoplasmic effector expression levels decreased dramatically to uniform low levels throughout the entire invasive hypha. Thus, there appears to be a lower constitutive level of PWL2 expression with upregulation during the earliest stage of biotrophic invasion. Our current working model is that cytoplasmic effector genes, encoding effector proteins destined for delivery to the rice cytoplasm, are 
highly up-regulated in the BIC-associated invasive hyphal cells and that this up-regulation is important for preferential BIC localization and delivery to the rice cytoplasm.

\section{References:}

[1] Khang CH, Berruyer R, Giraldo MC, et al, The Plant Cell 22 (2010), pp.1388-1403.

[2] Valent B, Khang CH, Current Opinion in Plant Biology 13 (2010), pp. 434-441

[3] The authors acknowledge funding from the USDA National Institute of Food and Agriculture, the National Research Initiative Competitive Grants Program, Grant Number 2010-65108-20538.

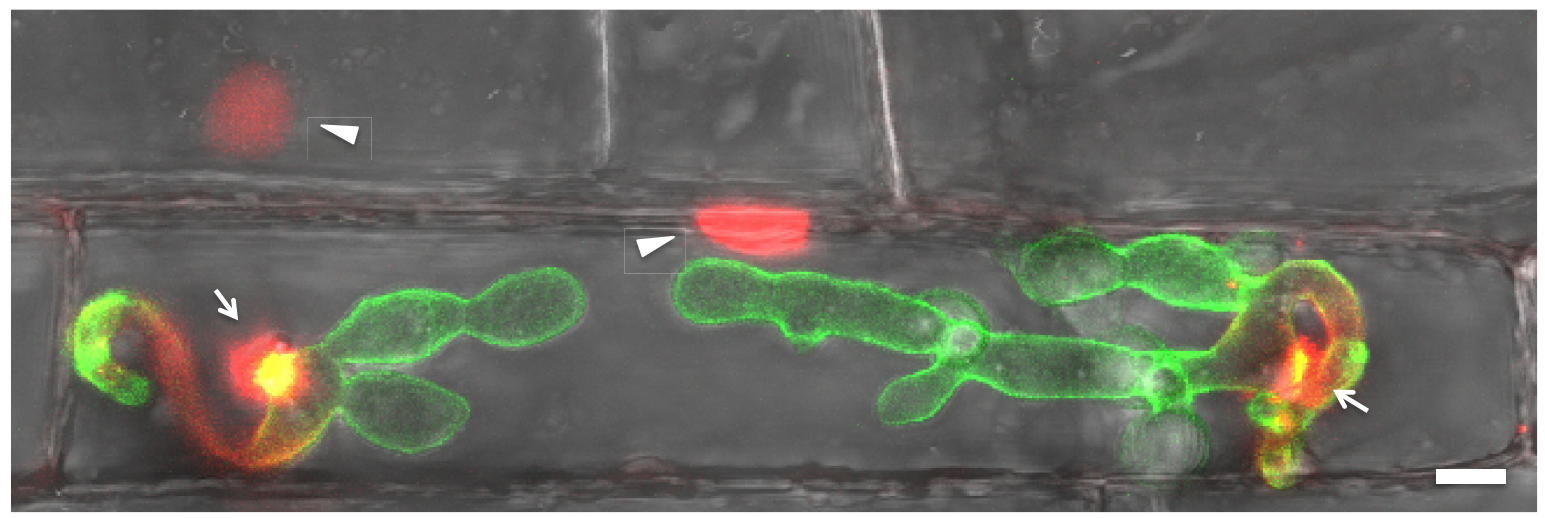

Figure 1. Projected confocal image of the rice blast fungus secreting the cytoplasmic effector protein Pwl2 linked to a red fluorescent protein and a nuclear localization signal. Two hyphae have invaded the same rice sheath epidermal cell at 28 hours post inoculation. The fungus also secreted the apoplastic effector protein Bas4 linked to green fluorescent protein, seen outlining the hyphae. Pwl2 preferentially accumulated in BICs (yellow from overlapping red and green proteins surrounded by red alone). Pwl2, but not Bas4, was translocated into the rice cytoplasm, shown by the red signal concentrated in the host nucleus using the linked nuclear localization signal. After reaching the rice cytoplasm, Pwl 2 moved ahead into adjoining uninvaded rice cells, suggesting that fungal effectors are sent ahead to prepare rice cells before fungal entry. Arrows $=$ BICs. Arrowheads $=$ rice nuclei. Bar $=5 \mu \mathrm{m}$

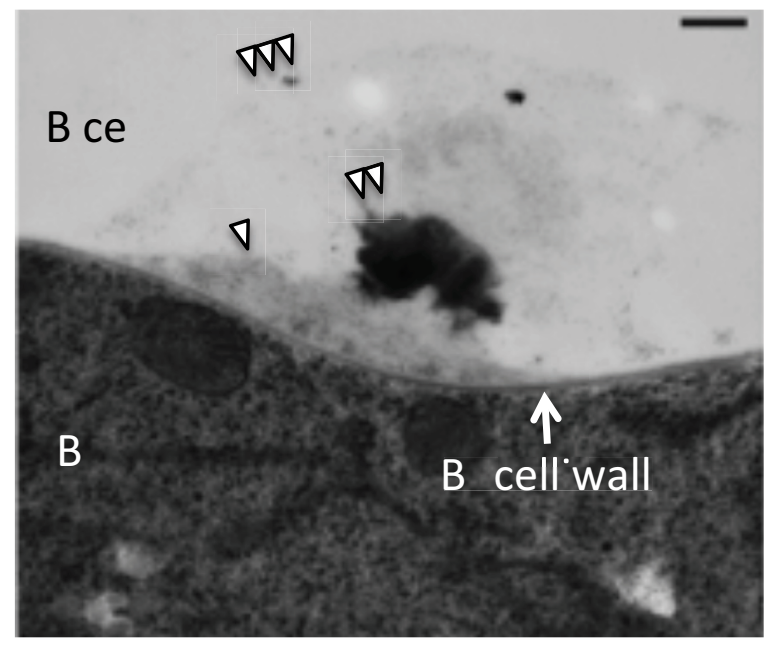

Figure 2. BIC ultrastructure. Transmission electron microscopy of infected rice tissue prepared using High Pressure Freezing-Freeze Substitution. Single arrowhead = laminar organization; double arrowhead $=$ lipid-rich region; triple arrowhead $=$ putative membrane cap that appears to encapsulate inner BIC structures. $\mathrm{IH}=$ invasive hypha. Bar $=0.3 \mu \mathrm{m}$. Sampled at 28 hours post inoculation. 